\title{
Enhanced Thermoelectric Performance and Lifetime in Acid-Doped PEDOT:PSS Films via Work Function Modification
}

\author{
Diego Rosas Villalva, Md Azimul Haque, Mohamad Insan Nugraha, Derya Baran* \\ King Abdullah University of Science and Technology (KAUST), Physical Sciences and \\ Engineering Division, KAUST Solar Center (KSC), Thuwal 23955-6900, Saudi Arabia. \\ E-mail address: Derya.baran@kaust.edu.sa
}




\begin{abstract}
In recent years, most of the work on p-type organic thermoelectrics focus on improving the thermoelectric properties of PEDOT:PSS through a sequential doping-dedoping process. However, the air-stability of thermoelectric parameters of these systems, which is essential for the realization of reliable devices remains largely unexplored. In this study, Poly (ethyleneimine)-ethoxylate (PEIE) acts as a work function modification agent and encapsulation layer to improve the thermoelectric performance and air-stability of nitric acid $\left(\mathrm{HNO}_{3}\right)$ doped PEDOT:PSS films. The evaporation of $\mathrm{HNO}_{3}$ is responsible for a simultaneous decrease in electrical conductivity and an increase in the Seebeck coefficient leading to the degradation of the power factor. PEIE reduces the evaporation of $\mathrm{HNO}_{3}$ from PEDOT:PSS, and increases the power factor from 72 to $168 \mu \mathrm{W} \mathrm{m} \mathrm{m}^{-1} \mathrm{~K}^{-2}$. After a week of exposure to air, the films show a power factor of $124 \mu \mathrm{W} \mathrm{m} \mathrm{m}^{-1} \mathrm{~K}^{-2}$, retaining $74 \%$ of its initial thermoelectric merits. These results underscore the importance of PEIE as a material for enhancing thermoelectric performance and air-stability in the development of polymer-based thermoelectrics.
\end{abstract}

KEYWORDS: Thermoelectrics, PEDOT:PSS, doping, stability, PEIE 


\section{INTRODUCTION}

Organic semiconductors (OSCs) for thermoelectric applications have recently gained significant interest in the materials research community. ${ }^{1-5}$ Unlike their inorganic counterparts, OSCs possess intrinsic low thermal conductivity and flexibility; they can be processed in solution, making them suitable for ink-jet, roll-to-roll, and 3D printing, and show good response even at room temperature. ${ }^{6}$ Furthermore, their structure can be designed in a modular way allowing versatility to tune their electronic, mechanical, and rheological properties. This makes them attractive for applications in wearable self-powered devices that are capable of utilizing body heat as an energy source. ${ }^{7,8}$ Thus, a steady and quick rise in the performance of OSCs has been observed during the past decade. This performance is mainly characterized through the dimensionless figure of merit $Z T=T \sigma S^{2} / \kappa$, where $\sigma$ is the electrical conductivity, $S$ is the Seebeck coefficient, $\kappa$ is the thermal conductivity, and $T$ is temperature. Due to their inherent low $\kappa$, most of the work in OSCs is driven by maximization the power factor $\left(P F=\sigma \mathrm{S}^{2}\right)$, typically achieved by doping. ${ }^{9}$

Amongst organic thermoelectric materials (OTEs), Poly(3,4-ethylene dioxythiophene):Poly(styrenesulfonate) is one of the best and most versatile p-type semiconductors due to its ease of processing (being water-dispersible) and tunable performance through doping. ${ }^{4,10}$ Several works carried out to date on PEDOT:PSS focus on finding an optimal ratio between $\sigma$ and $S$ to maximize the $P F$ through sequential doping - de-doping process. ${ }^{11-14}$ This is done by casting a film of PEDOT:PSS followed by exposure to an oxidizing agent, typically a strong acid, leading to enhancement of $\sigma$ (up to $5400 \mathrm{~S} \mathrm{~cm}^{-1}$ ) due to the removal of the non-conductive PSS and increased crystallinity. ${ }^{12,15-18}$ Common dopants include ethylene 
glycol, DMSO, methanol, and different acids such as formic acid, and sulfuric acid $\left(\mathrm{H}_{2} \mathrm{SO}_{4}\right) \cdot{ }^{12,19,20}$ In a second step, PEDOT is treated with an electron-donating group such as $\mathrm{NaOH}$, hydrazine, or TDAE to de-dope it and thus increase the Seebeck coefficient. ${ }^{11,21,22}$ Upon optimization of the ratio of the oxidizing and reducing agents, $Z T \mathrm{~s}$ as high as 0.42 have been achieved..$^{18}$ Nonetheless, the degradation and stability of p-type doped organic semiconductors have received limited attention. ${ }^{19,23-27}$

Although p-type organic semiconductors are generally regarded as air-stable, there are a few degradation pathways such as water absorption and diffusion of molecules with small sizes or low boiling point, which leads to the creation of trap states or morphological changes in the microstructure of the OSC. ${ }^{28-30}$ Formic acid (FA) is a common dopant for PEDOT:PSS, which makes a clear example of such a mechanism. Its low boiling point leads to evaporation of the FA and thus losses in electrical conductivity. Nitric acid $\left(\mathrm{HNO}_{3}\right)$ and $\mathrm{H}_{2} \mathrm{SO}_{4}$, are some of the dopants capable of achieving metallic conductivities i.e. as high as $4000 \mathrm{~S} \mathrm{~cm}^{-1} \cdot{ }^{15}$ However, the low boiling point of $\mathrm{HNO}_{3}\left(80{ }^{\circ} \mathrm{C}\right)$ poses a threat to the stability and lifetime of thermoelectric devices as, similarly to formic acid, it might evaporate from the PEDOT film, leading to changes in electronic properties. ${ }^{19}$ Previous works have shown that Poly (ethyleneimine) ethoxylated (PEIE) can be used to coat N-type thermoelectric films based on carbon nanotubes, producing a doping effect and enhanced stability, thanks to its impermeability to oxygen. ${ }^{31}$ Other reports have shown that a very thin layer of this polymer can lower the work function of PEDOT transport layers in solar cells. ${ }^{32,33}$ Additionally, it has been shown that it can lead to enhanced mobility in N-type organic field-effect transistors by lowering the work function of the metallic contacts, this has been shown to occur thanks to the molecular dipole moments of the 
ethyleneamine and ethoxy groups. ${ }^{34}$ Despite the insulating nature of PEIE, previous studies show that charge injection can occur through tunneling and thermionic emission which makes it an interesting material to be studied as a layer for contact engineering and stability enhancement in thermoelectric materials.

In this work, we developed a coating based on PEIE for both work function modification and in-air-stability enhancement of organic thermoelectrics. $\mathrm{HNO}_{3}$ doped PEDOT:PSS films were used as a model to develop and optimize our PEIE layer. The power factor is improved from 72 $\mu \mathrm{W} \mathrm{m}{ }^{-1} \mathrm{~K}^{-2}$ in films only doped with $\mathrm{HNO}_{3}$ to $168 \mu \mathrm{W} \mathrm{m}{ }^{-1} \mathrm{~K}^{-2}$ upon coating with PEIE. After exposing the samples for one week to air, the power factor of the PEIE coated films lost only 26\%. Furthermore, we showed that the PEIE coating is also compatible with $\mathrm{H}_{2} \mathrm{SO}_{4}$ hence making PEIE a potential universal method to improve the stability of OTEs.

\section{EXPERIMENTAL}

Materials: PEDOT:PSS (PH1000) was obtained from Heraeus, Nitric acid 70\%, N, Ndimethylformamide 99.8\%, and Polyethyleneimine, 80\% ethoxylated (aqueous solution 37 wt.\%) were obtained in from Sigma-Aldrich.

\section{Film preparation}

Soda-lime glass substrates $(500 \mu \mathrm{m}$ thick) were cut into $2 \times 2 \mathrm{~cm}$ squares and cleaned through sonication in DI water, acetone, and isopropanol; finally they were treated with UV-O3 for 15 min. PH1000 was filtered using a nylon filter with a pore size of $200 \mathrm{~nm}$ and then spin-coated on top of the soda-lime glass substrates at $2000 \mathrm{RPM}$ for $60 \mathrm{~s}$ and annealed at $140{ }^{\circ} \mathrm{C}$ for $10 \mathrm{~min}$. The resulting films were then treated with $\mathrm{HNO}_{3}(250 \mu \mathrm{L})$ dropped on top of them and letting 
rest for $15 \mathrm{~min}$. The films were then dried using a $\mathrm{N}$ gun. Afterward, PEIE solutions in DMF:H2O 4:1 with concentrations ranging from 0.2 to $1.2 \mathrm{wt} . \%$, prepared one day in advance, were spin-coated on top of the $\mathrm{HNO}_{3}$ treated PH1000 films at 5000 RPM for $60 \mathrm{~s}$, the resulting films were annealed at $80 \mathrm{C}$ for $2 \mathrm{~min}$.

\section{Characterization}

A home-made set-up consisted of two Peltier devices, 2 temperature sensors (sensirion SHTC1), and two Keithley SMUs (2420 source-meter and 2182 Nanovoltmeter), was used to measure thermo-voltage and thus calculate Seebeck coefficient. We used a constantan sample as reference calibration for our system and obtained $\mathrm{S}=-39 \pm 2 \mu \mathrm{V} \mathrm{K} \mathrm{K}^{-1}$ to validating the reliability of our setup. Conductivity was determined through the 4 point probe method. The evolution of the thermoelectric properties was recorded for one week keeping samples both in air and inside a $\mathrm{N}_{2}$ filled glovebox.

UV-Vis-NIR spectra were recorded using Cary 5000 spectrophotometer from Agilent spectroscopy, Raman spectroscopy was done using NTEGRA spectra by NT-MDT. Morphologic characterization and film thickness were measured using atomic force microscopy (SolverNext by NT-MDT). Charge carrier concentration was determined using Hall Effect measurement system (Lake Shore 8400 series). PESA (Photoelectron Spectroscopy in Air, Riken Keiki model AC-2) was used to determine the work function. Grazing Incidence X-ray Diffraction (GIXRD) was performed using a Bruker D8 Advance with Davinci design.

\section{RESULTS AND DISCUSSION}

PEDOT:PSS films were spin-coated on glass using CLEVIOS ${ }^{\mathrm{TM}}$ PH1000 from Heraeus and 
sequentially doped with $\mathrm{HNO}_{3}$ to obtain $\sigma$ up to $4000 \mathrm{~S} \mathrm{~cm}^{-1}$ The high conductivity is attributed in the first place, to the removal of the insulating PSS- which is confirmed by the removal of two characteristic peaks from the UV absorption spectra (Figure S1) and Raman spectra by the removal of the peak at $1600 \mathrm{~cm}^{-1}$. In the second place, the removal of PSS enhances the packing of PEDOT, as seen in GIXRD (Figure S2). In a second step, a layer of PEIE was spin-coated on top of these films, the thickness of this layer was adjusted by varying the concentration of PEIE in the coating solution as shown in Figure 1a.

a)
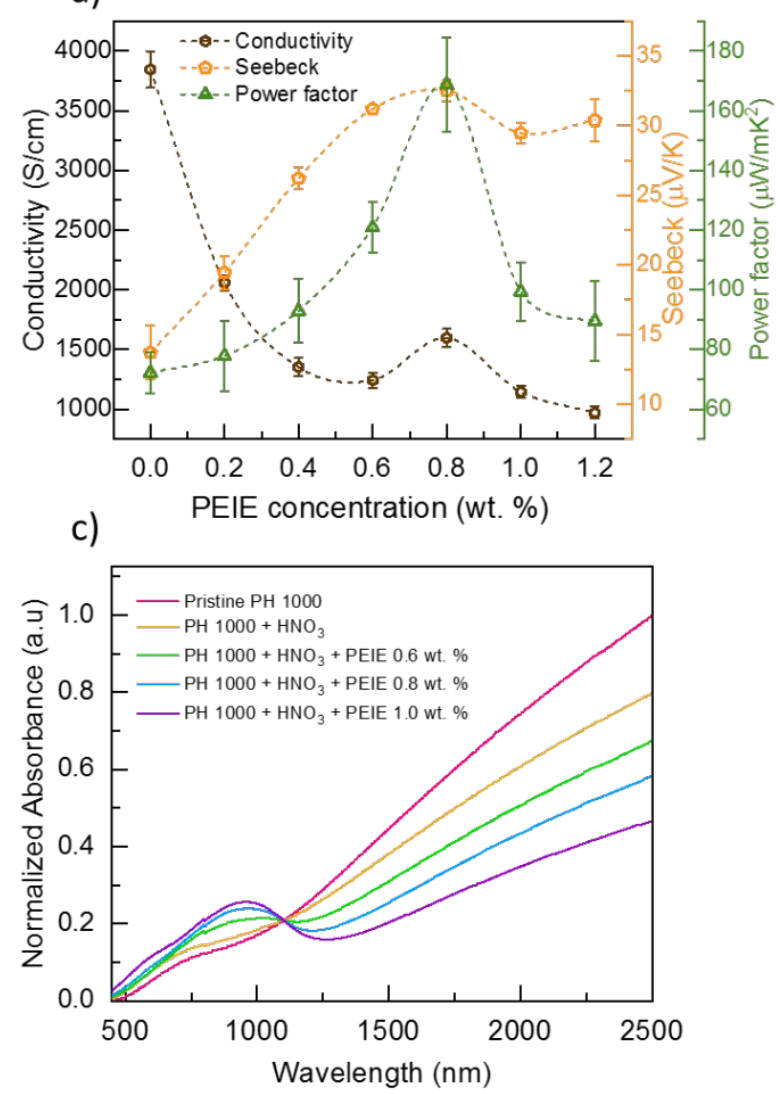

b)

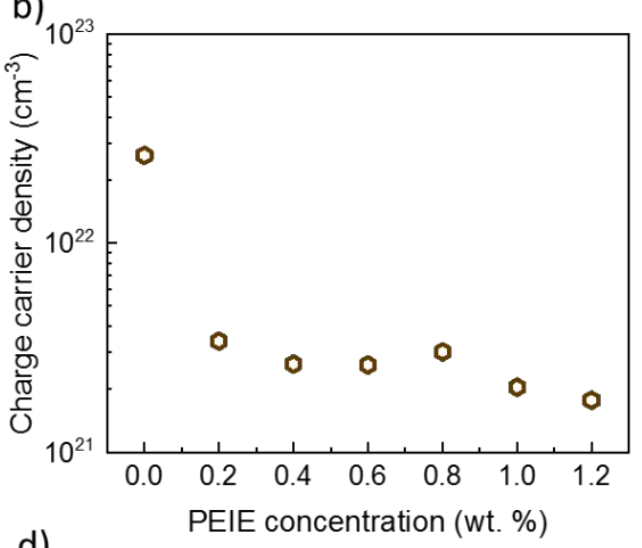

d)

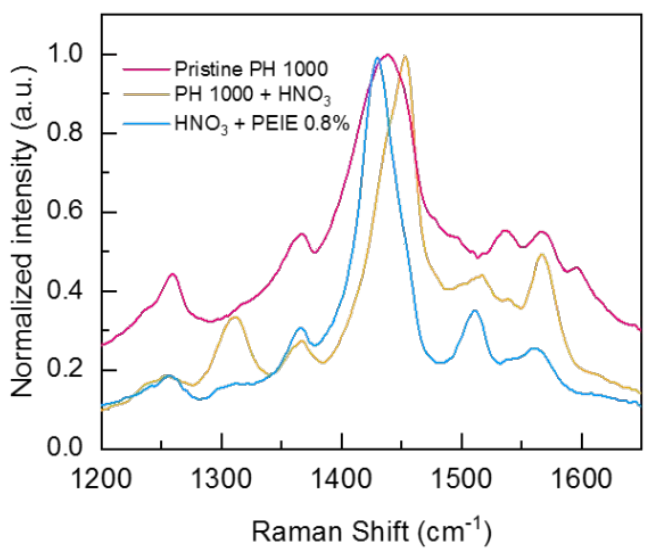

Figure 1. a) Change of thermoelectric properties of $\mathrm{HNO}_{3}$ doped PEDOT:PSS and b) charge carrier density (n) upon coating PEIE from solutions with different concentrations. c) Absorption spectroscopy d) Raman spectroscopy. 
The maximum power factor $\left(168.74 \mu \mathrm{W} \mathrm{m} \mathrm{m}^{-1} \mathrm{~K}^{-2}\right)$ is obtained when a solution with $0.8 \mathrm{wt} \%$ is used to coat the $\mathrm{HNO}_{3}$ doped PEDOT:PSS. $S$ and $\sigma$ are $32 \mu \mathrm{V} \mathrm{k}^{-1}$ and $1595 \mathrm{~S} \mathrm{~cm}^{-1}$ respectively. Measuring thermal conductivity $(\kappa)$ is rather challenging for OSC thin films. This material shows anisotropic $\kappa$, leading to different in-plane $(\kappa \|)$ and out-of-plane $(\kappa \perp)$. However, previous studies on systems based on doped PEDOT:PSS report approximate $\kappa$ of $0.25 \mathrm{~W} \mathrm{~m}^{-1} \mathrm{~K}^{-1} .35-37$ Applying this value, we approximate a ZT of 0.2 for our system. By increasing the PEIE wt. \% a thicker layer is formed, and the electron-donating character of the ammine groups contribute to reducing PEDOT:PSS and thus enhance $S$ while decreasing $\sigma$. Hall effect characterization (Figure 1b) shows a high charge carrier density $(n)$ for $\mathrm{HNO}_{3}$ doped PEDOT which decreases upon addition of higher amounts of PEIE, suggesting a dedoping process.

Further insight into the effect of PEIE is provided by absorption and Raman spectroscopy (Figures 1c, d). Absorption spectra show a clear correlation between the increasing amount of PEIE and the decrease in the absorbance of the bipolaron band (broad feature in the NIR region) For Raman spectroscopy, it is possible to correlate the degree of oxidation to the thiophene ring symmetric stretching at $1444 \mathrm{~cm}^{-1}$. Higher oxidation shifts the peak to higher wavenumbers and reduction has the opposite effect. For this reason, $\mathrm{HNO}_{3}$ doped PEDOT show a narrow peak shifted towards $1450 \mathrm{~cm}^{-1}$ (oxidation). Spin coating PEIE on top leads to further narrowing of the peak and shifting towards $1430 \mathrm{~cm}^{-1}$ (Reduction). Another important evolution of peaks takes place in the region between 1500 and $1600 \mathrm{~cm}^{-1}$. The fully oxidized PEDOT shows a weak peak at $1517 \mathrm{~cm}^{-1}$ and an intense peak at $1570 \mathrm{~cm}^{-1}$. Upon application of the PEIE layer, the relative intensities of these peaks are reversed i.e. the peak at $1717 \mathrm{~cm}^{-1}$ becomes more intense and the one at 1570 becomes weaker and shifts to $1555 \mathrm{~cm}^{-1}$. This region is associated with the 
symmetric and antisymmetric stretching of the thiophene ring, suggesting interconversion between quinoid and aromatic structures of PEDOT. ${ }^{38}$ Finally, the peak at $1315 \mathrm{~cm}^{-1}$ is very intense in the sample with no PEIE and gradually decreased upon the addition of PEIE. This peak is not characteristic of PEDOT and is instead attributed to the presence of $\mathrm{HNO}_{3} \cdot{ }^{39}$ The observed changes are compliant with previous reports of doped and de-doped PEDOT:PSS and can help in tracking and confirming the doping/de-doping of PEDOT by $\mathrm{HNO}_{3}$ and PEIE. ${ }^{13}$ This can be further confirmed by absorption spectroscopy (Figure S3), which is normalized using the isosbestic point of PEDOT. Increasing the concentration of PEIE leads to the reduction of the bipolaron band above $1250 \mathrm{~nm}$ and increases the polaron band (1550 nm). In the more reduced samples, it is possible to observe already the $\pi \rightarrow \pi^{*}$ transition close to $600 \mathrm{~nm} .^{21}$
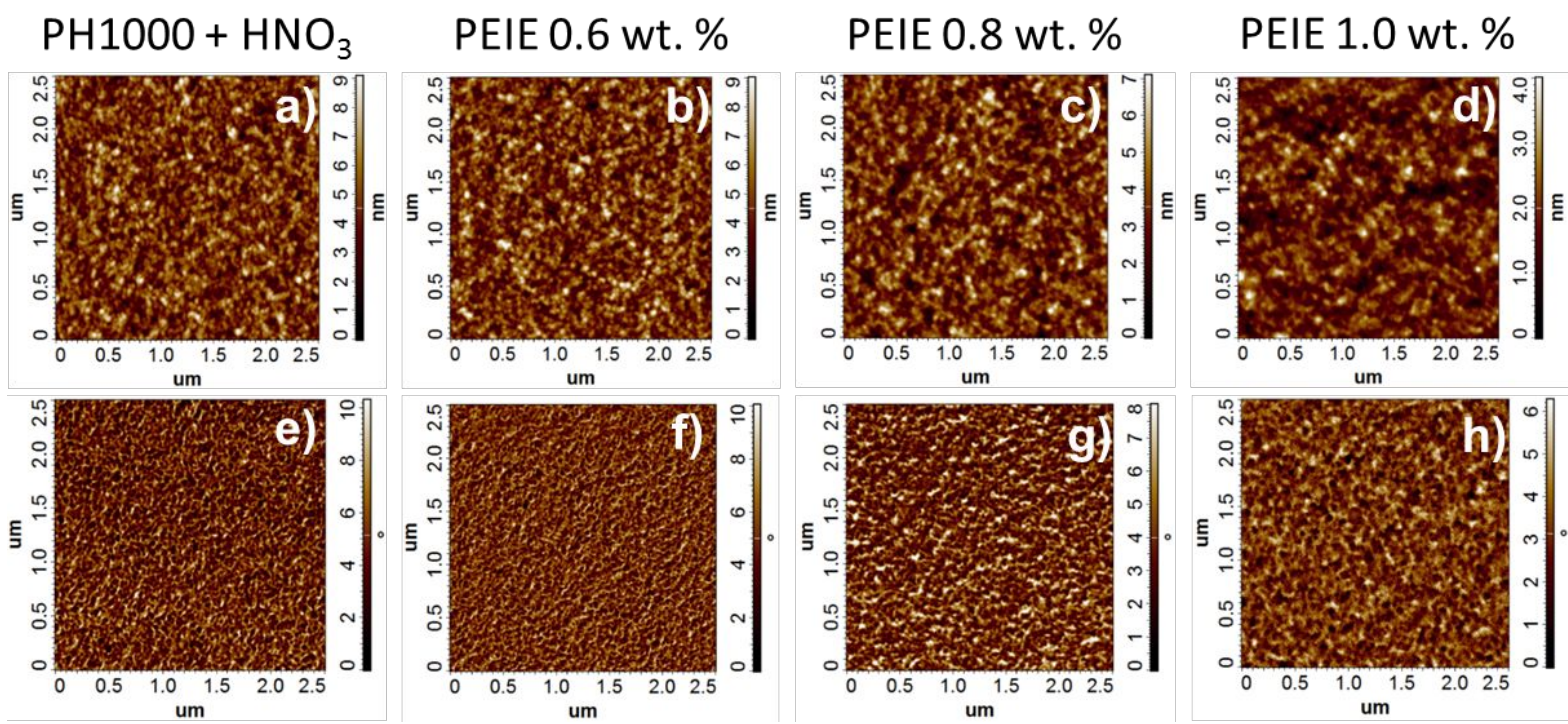

Figure 2. AFM topography of PEDOT films without PEIE (a, e) and increasing ratios of PEIE. $(\mathrm{a}-\mathrm{d})$ and phase $(\mathrm{e}-\mathrm{h})$ images. Summary in Table $\mathbf{S 1}$.

We used Photo-Electron Spectroscopy in Air (PESA) to determine the work function (Figure S4) and atomic force microscopy (AFM) to track the morphological changes in the films. The results from PESA show that the work function increases upon increasing the PEIE 
concentration, reaching the maximum at $1.0 \mathrm{wt}$. $\%$, nevertheless the sample with 0.8 wt. $\%$ shows better performance. Previous reports show that, despite the insulating nature of PEIE, it can be used as an interfacial layer to facilitate charge carrier extraction by tunneling. ${ }^{40}$ The observed changes in the work function of PEDOT can be attributed to the dipole moment of the ethoxy groups contained in PEIE. Previous reports suggest these groups as the main contributor for the change of work function in other OSCs. ${ }^{41}$ We hypothesize that the formation of a continuous layer of PEIE, with the optimal thickness for carrier extraction, takes place when we use the solution with PEIE 0.8 wt. \%, and therefore a higher conductivity is observed for this sample. This is supported by AFM characterization (Figure 2). For films spin-coated from solutions with content lower than 0.8 wt.\% PEIE, the morphological changes are minimal (i.e. compared to the sample with no PEIE). In contrast, for 0.8 wt.\% PEIE (Figure $2 \mathbf{c}$ and $\mathbf{g}$ ) the roughness is decreased from 1.36 to $1.08 \mathrm{RMS}$ and the thickness is increased from $25-34 \mathrm{~nm}$ to 30-36 nm providing evidence for the formation of a continuous layer of PEIE. Using a solution with a slightly higher PEIE content (1.0 wt. \%) has an even more dramatic decrease in film roughness $(0.586 \mathrm{RMS})$ and increment of the thickness $(37-45 \mathrm{~nm})$ suggesting that the PEIE layer is too thick to allow efficient charge carrier extraction. 

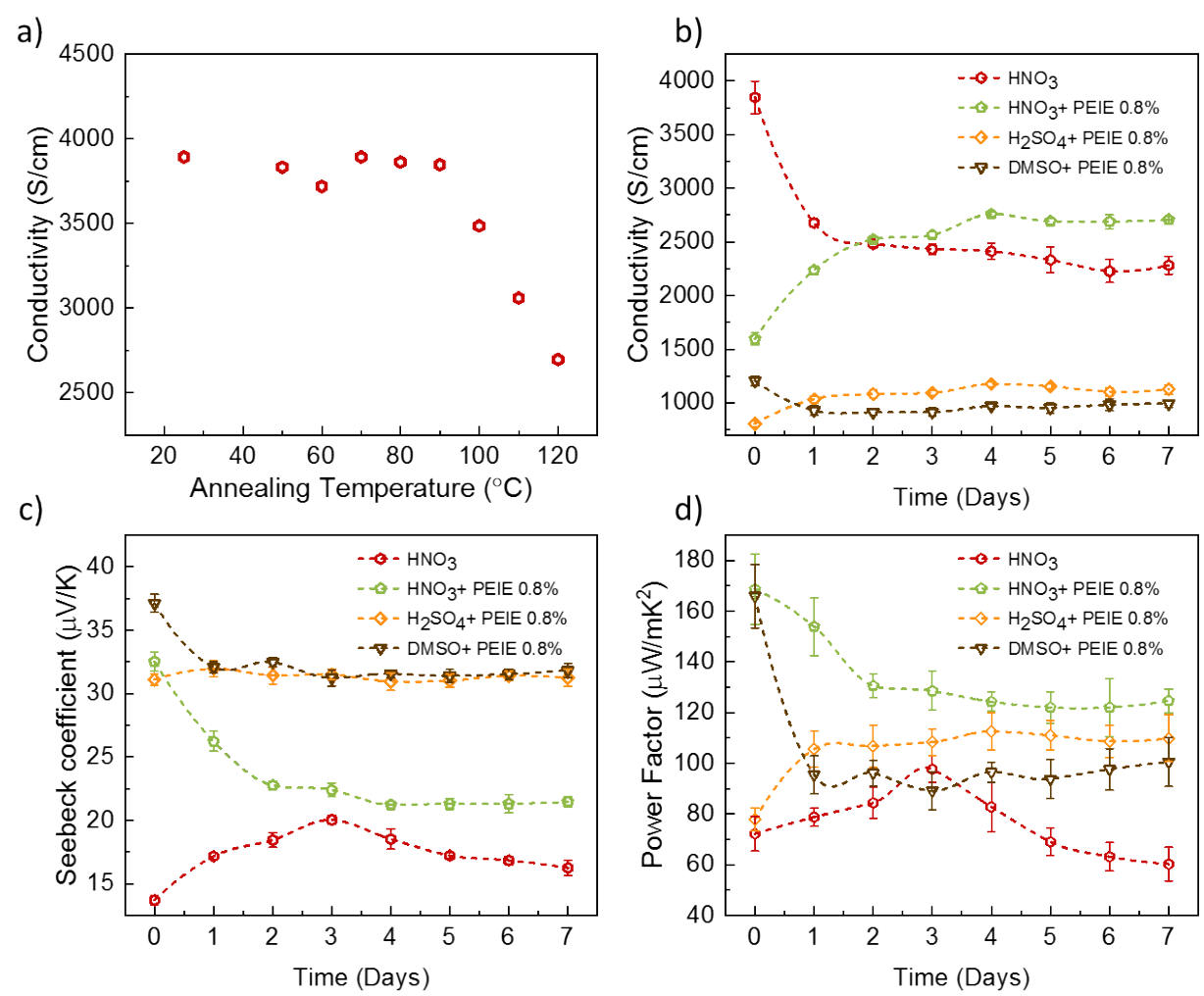

Figure 3. Stability of the thermoelectric properties. a) Thermal degradation of electrical conductivity $(\boldsymbol{\sigma})$ in $\mathrm{PH} 1000+\mathrm{HNO}_{3}$ b) Variation of $\boldsymbol{\sigma}$ c) Seebeck coefficient and d) Power Factor upon exposure to air of PEDOT samples with different treatments

After optimizing the thickness of the PEIE layer, we tested in-air-stability of the PEDOT sample doped only with $\mathrm{HNO}_{3}$ to serve as control, and compare it to that of the sample coated with 0.8 wt. $\%$ PEIE. Two more samples where $\mathrm{HNO}_{3}$ is replaced by $\mathrm{H}_{2} \mathrm{SO}_{4}$ or DMSO were prepared and coated with the same solution of PEIE to test the compatibility of this method with other dopants. As mentioned before, one of the main concerns of doping with $\mathrm{HNO}_{3}$ is its low boiling point, as it can evaporate and lead to losses in conductivity. This effect is shown by annealing an $\mathrm{HNO}_{3}$ doped PEDOT sample at different temperatures (Figure 3a). The conductivity remains relatively stable until around $90{ }^{\circ} \mathrm{C}$, afterward, the conductivity starts dropping quickly due to the evaporation of nitric acid. A similar effect occurs when the sample is 
aged either in air (Figures $3 \mathbf{b}-\mathbf{d}$ ), or inside a $\mathrm{N}_{2}$ filled glovebox (Figures S5). On day 1 after doping, the conductivity decreases from $3800 \mathrm{~S} / \mathrm{cm}$ to $2400 \mathrm{~S} / \mathrm{cm}$. Consequently, $S$ slightly increases due to the lower oxidation degree of PEDOT upon $\mathrm{HNO}_{3}$ evaporation, reaching a maximum on day $3\left(20 \mu \mathrm{V} \mathrm{k}^{-1}\right)$ and maximum $\mathrm{PF}$ of $97.62 \mu \mathrm{W} \mathrm{m} \mathrm{m}^{-1} \mathrm{~K}^{-2}$. On day $4, \mathrm{~S}$ starts decreasing along with the power factor, leading to an overall decrease in the performance.

In contrast, a significant improvement of the stability in air is observed after coating PEIE on top of the doped PEDOT films. In the case of the treatment with $\mathrm{HNO}_{3}+\mathrm{PEIE}$, we observe an opposite trend in the electrical conductivity i.e. increasing conductivity as the sample ages and stabilization on day 5 at $2700 \mathrm{~S} / \mathrm{cm}$. In terms of $S$, the value drops from $32.52 \mu \mathrm{V} \mathrm{k}^{-1}$ on day 0 to about $21.3 \mu \mathrm{V} \mathrm{k}^{-1}$ (Figure 3c), which is consistent with an inverse relationship between $\sigma$ and $\mathrm{S}$. Despite the drop in $\mathrm{S}$, the performance is higher in comparison with the samples treated only with $\mathrm{HNO}_{3}$. This is easily observed by comparing the $P F$ of both samples, i.e. $122 \mu \mathrm{W} \mathrm{m}^{-1} \mathrm{~K}^{-2}$ as observed after seven days, which is two times higher than that of the sample treated only with nitric acid $\left(60.22 \mu \mathrm{W} \mathrm{m}{ }^{-1} \mathrm{~K}^{-2}\right.$ after 7 days). In the case of $\mathrm{H}_{2} \mathrm{SO}_{4}$, the behavior remains relatively constant throughout the study, possibly due to the higher boiling point of this acid, and further improvements in the PF might be achieved by additional optimization. Finally, in the case of DMSO doped PEDOT we observed a drop in power factor of about $50 \%$ from day 0 to day 1 , suggesting that the PEIE coating might be more suitable for acid doping, where the doping occurs by protonation and displacement of the $\mathrm{PSS}^{-}$anion, $\mathrm{NO}_{3}^{-}$in our case, which helps in the stabilization of cations in the backbone of PEDOT. ${ }^{42-44}$ On the other hand, DMSO dopes and stabilizes charges in PEDOT owing to its strong dipole moment, which screens charges from PSS-, similar to dimethyl formamide (DMF), ethyleneglycol (EG), or Poly(Ethylene Glycol) 
(PEG). ${ }^{45-48}$ This suggests that PEIE coating might be less effective with such solvents.

Previous reports show that poly (ethyleneimine) (PEI), the non-ethoxylated version of PEIE, can form a protective barrier for n-type thermoelectric carbon nanotubes that prevents oxygen permeation and diffusion into the material. ${ }^{31}$ Similarly, we think that the layer of PEIE on top of treated PEDOT:PSS films, prevents the full evaporation of $\mathrm{HNO}_{3}$. This, however, does not fully explain the strange trend observed in conductivity (i.e. increasing a few days after PEIE application). Our theory is that the thermal annealing carried out during coating with PEIE promotes $\mathrm{HNO}_{3}$ diffusion out of PEDOT. The layer of PEIE prevents the escape of the acid and in the subsequent days, it migrates back to PEDOT oxidizing it again, thus increasing its conductivity. This is consistent with the data collected from Raman spectroscopy. Finally, to discard the possibility of oxygen being the culprit for the changes we observed, we kept a set of samples inside a $\mathrm{N}_{2}$ filled glovebox with a pressure of $4 \mathrm{~atm}$. The observed trend was similar to that of the samples kept in air (Figure S4).

To understand the degradation of $\mathrm{HNO}_{3}$ doped PEDOT, we used Raman spectroscopy (Figure 4a). As mentioned before, the peak at $1315 \mathrm{~cm}^{-1}$ is associated with the presence of nitric acid in the films. On day 0 this peak shows a high intensity in comparison to its neighboring peaks which quickly drops on day 1 confirming the evaporation of $\mathrm{HNO}_{3}$. Simultaneously, we observe a shift in the main peak from 1450 to $1430 \mathrm{~cm}^{-1}$, as well as variations in the relative intensity of the peaks between 1500 and $1600 \mathrm{~cm}^{-1}$, which are associated with the symmetric and antisymmetric stretching of the thiophene ring. This is congruent with a transition from the fully oxidized and highly conductive state of PEDOT (bipolaron), to a reduced and less conductive state (polaron). ${ }^{38}$ On the other hand, the sample coated with PEIE (Figure 4b) starts in a 
relatively reduced state on day 0 and quickly transitions to a more oxidized state in the following days, as the peak at $1512 \mathrm{~cm}^{-1}$ decreases with respect to the peak at $1565 \mathrm{~cm}^{-1}$. This change correlates well with the changes observed in $\sigma$ for this sample (figure $\mathbf{3 b}$ ).

a)

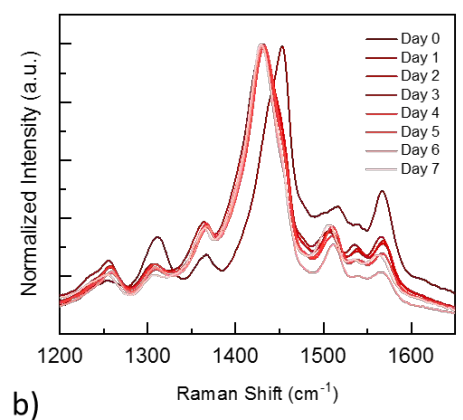

b)

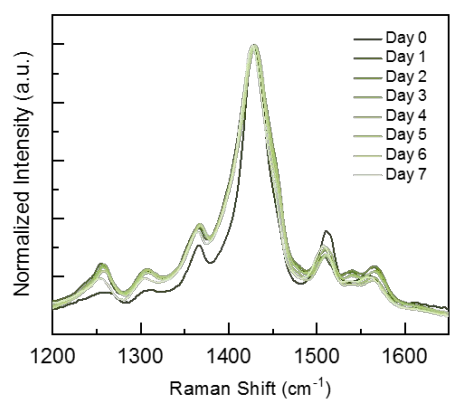

c)

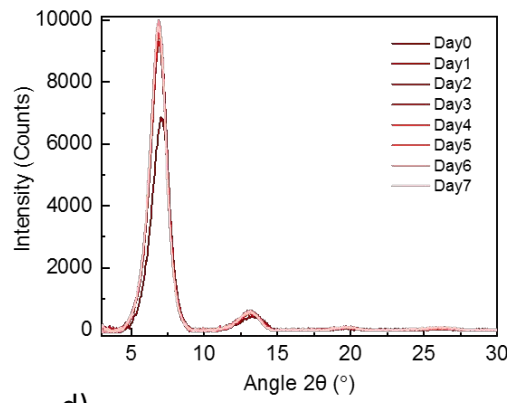

d)

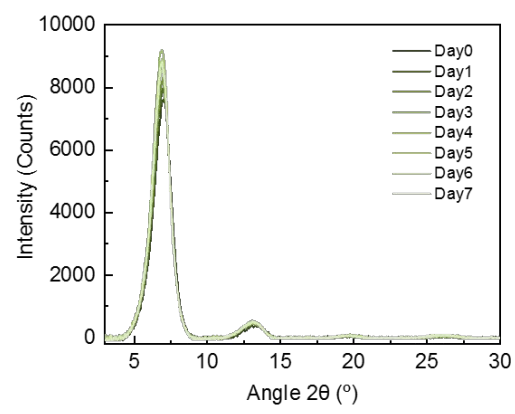

e)

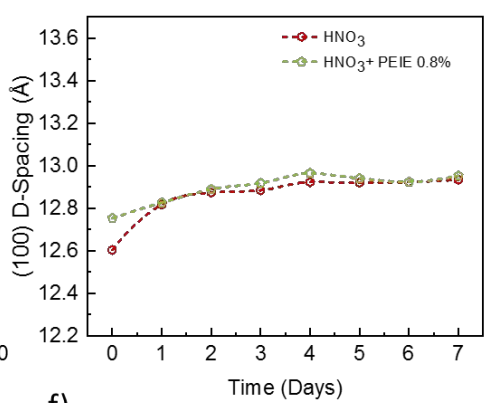

f)

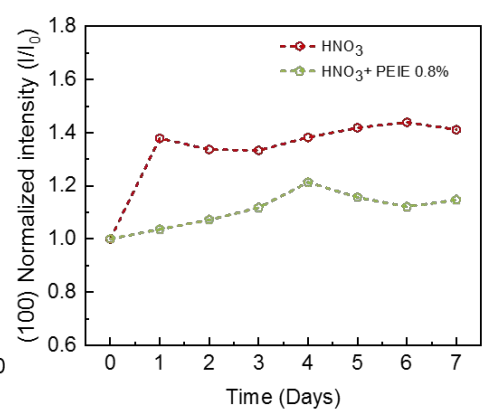

Figure 4. Comparison of the time stability of $\mathrm{HNO}_{3}$ doped PEDOT:PSS samples with (green) and without (Red) PEIE. a) Raman spectra for PEDOT:PSS $+\mathrm{HNO}_{3}$ and b) PEDOT:PSS + $\mathrm{HNO}_{3}+$ PEIE layer. c) GIXRD for PEDOT:PSS $+\mathrm{HNO}_{3}$ and d) GIXRD for PEDOT:PSS + $\mathrm{HNO}_{3}+$ PEIE. e) d-spacing and f) relative intensity comparisons from GIXRD.

To verify if there were any structural changes in these films with time, we performed Grazing Incident X-Ray Diffraction (GIXRD). It is possible to observe variations for both intensity and interplanar distance of the lamellar stacking, particularly in the (100) plane for PEDOT. The sample treated only with $\mathrm{HNO}_{3}$ shows an increment in D-spacing from 12.6 to $12.9 \AA$ (Figure 4c), calculated using the central $2 \theta$ value from the full width at half maximum (FWHM). This change is less drastic for the samples coated with PEIE (Figure 4d) (between 12.8 and $12.9 \AA$ A). 
It seems contradictory that the evaporation of $\mathrm{HNO}_{3}$ would lead to increased lamellar spacing, but we suggest that the presence of the $\mathrm{NO}_{3}{ }^{-}$anion reduces the lamellar spacing by stabilization of the positive charges in the backbone of PEDOT. After $\mathrm{NO}_{3}{ }^{-}$, evaporates these positive charges experience repulsive forces that lead to the observed increment in lamellar spacing. Similar effects have been observed for the $\pi \rightarrow \pi$ stacking in poly (3-hexylthiophene) (P3HT) doped with $\mathrm{F}_{4} \mathrm{TCNQ}$ and molybdenum tris[1-(methoxycarbonyl)-2-(trifluoromethyl)-ethane-1,2-dithiolene] $\left(\mathrm{Mo}\left(\mathrm{tfd}-\mathrm{CO}_{2} \mathrm{Me}\right)_{3}\right) \cdot{ }^{49}$ Changes in intensity are associated with preferential growth or stacking along a particular direction. In this case, the higher intensity for the (100) plane represents a preferential orientation along the [100] direction. For PEDOT without PEIE, the increment in intensity is about $40 \%$ compared to day 0 , whereas in the case of PEIE coated PEDOT the increment is only about $15 \%$. A possible explanation to this is that the evaporation of $\mathrm{HNO}_{3}$ allows further stacking of PEDOT along this direction leading to the observed decrement of the Seebeck coefficient, whereas in the case of the PEIE coated samples, the presence of $\mathrm{HNO}_{3}$ limits such structural change. This is supported by previous reports that introduced a formalism to describe the contributions to the Seebeck coefficient, which is a sum of a mechanismdependent transport entropy and vibrational entropy. ${ }^{50-52}$ Hence the higher crystalline ordering would decrease the vibrational entropy of our system, and then reduce the Seebeck coefficient with it. Although more extensive research on the dependency of $S$ on crystallinity is needed, we think that the lower ordering in the PEIE coated films might be the origin for a higher vibrational entropy and thus explain the higher S observed in the sample with 0.8 wt. \% PEIE. 


\title{
CONCLUSION
}

In this work, we explored the role of PEIE as an additional layer for acid doped PEDOT:PSS for the simultaneous improvement of thermoelectric properties and in-air-stability of OTEs. The ethoxy groups contained in PEIE enhance charge carrier extraction by modifying the work function of PEDOT, while the amino groups increase the Seebeck coefficient. Furthermore, this polymer provides mild encapsulation that prevents dopant evaporation, and thus escape of nitric acid leading to enhanced stability. This underlines the importance of work function modification layers as a method to improve the thermoelectric properties of semiconducting polymers. By optimizing the thickness of the PEIE layer we achieved a stable power factor of $124 \mu \mathrm{W} \mathrm{m} \mathrm{m}^{-1} \mathrm{~K}^{-2}$ which is twice that of the $\mathrm{HNO}_{3}$ doped films without PEIE. We believe that these findings are not only important for p-type thermoelectrics but are also relevant in the field of n-type organic semiconductors and the efforts in doping them, as PEIE can prevent oxygen permeation to the OSCs which is known to be a major issue for their stability in air.

\begin{abstract}
ASSOCIATED CONTENT
Supporting Information. UV-Vis, GIXRD, Raman, and PESA spectra are provided. This material is available free of charge via https://pubs.acs.org.
\end{abstract}

\section{Notes}

The authors declare no conflicts of interest

\section{AUTHOR INFORMATION}




\section{Corresponding Author}

*Derya Baran (derya.baran@kaust.edu.sa)

\section{Author Contributions}

The manuscript was written through the contributions of all authors. All authors have approved the final version of the manuscript.

\section{ACKNOWLEDGMENT}

This publication is based upon work supported by the King Abdullah University of Science and Technology (KAUST) Office of Sponsored Research (OSR) under Award No. OSR-CRG20183737.

\section{REFERENCES}

(1) Han, S.; Jiao, F.; Khan, Z. U.; Edberg, J.; Fabiano, S.; Crispin, X. Thermoelectric Polymer Aerogels for Pressure-Temperature Sensing Applications. Adv. Funct. Mater. 2017, 27, 1703549.

(2) Zhang, F.; Zang, Y.; Huang, D.; Di, C.; Zhu, D. Flexible and Self-Powered TemperaturePressure Dual-Parameter Sensors Using Microstructure-Frame-Supported Organic Thermoelectric Materials. Nat. Commun. 2015, 6, 8356.

(3) Bubnova, O.; Crispin, X. Towards Polymer-Based Organic Thermoelectric Generators. Energy Environ. Sci. 2012, 5, 9345-9362.

(4) Russ, B.; Glaudell, A.; Urban, J. J.; Chabinyc, M. L.; Segalman, R. A. Organic 
Thermoelectric Materials for Energy Harvesting and Temperature Control. Nat. Rev. Mater. 2016, 1,16050 .

(5) Campoy-Quiles, M. Will Organic Thermoelectrics Get Hot? Philos. Trans. R. Soc. A Math. Phys. Eng. Sci. 2019, 377, 20180352.

(6) Kee, S.; Haque, M. A.; Corzo, D.; Alshareef, H. N.; Baran, D. Self-Healing and Stretchable 3D-Printed Organic Thermoelectrics. Adv. Funct. Mater. 2019, 29, 1905426.

(7) Kee, S.; Kim, H.; Paleti, S. H. K.; El Labban, A.; Neophytou, M.; Emwas, A. H.; Alshareef, H. N.; Baran, D. Highly Stretchable and Air-Stable PEDOT:PSS/Ionic Liquid Composites for Efficient Organic Thermoelectrics. Chem. Mater. 2019, 31, 3519-3526.

(8) Park, T.; Park, C.; Kim, B.; Shin, H.; Kim, E. Flexible PEDOT Electrodes with Large Thermoelectric Power Factors to Generate Electricity by the Touch of Fingertips. Energy Environ. Sci. 2013, 6, 788-792.

(9) Beekman, M.; Morelli, D. T.; Nolas, G. S. Better Thermoelectrics through Glass-like Crystals. Nat. Mater. 2015, 14, 1182-1185.

(10) Zhang, Q.; Sun, Y.; Xu, W.; Zhu, D. Organic Thermoelectric Materials: Emerging Green Energy Materials Converting Heat to Electricity Directly and Efficiently. Adv. Mater. 2014, 26, $6829-6851$.

(11) Khan, Z. U.; Bubnova, O.; Jafari, M. J.; Brooke, R.; Liu, X.; Gabrielsson, R.; Ederth, T.; 
Evans, D. R.; Andreasen, J. W.; Fahlman, M.; Crispin, X. Acido-Basic Control of the Thermoelectric Properties of Poly(3,4-Ethylenedioxythiophene)Tosylate (PEDOT-Tos) Thin Films. J. Mater. Chem. C 2015, 3, 10616-10623.

(12) Kim, N.; Kee, S.; Lee, S. H.; Lee, B. H.; Kahng, Y. H.; Jo, Y. R.; Kim, B. J.; Lee, K. Highly Conductive PEDOT:PSS Nanofibrils Induced by Solution-Processed Crystallization. Adv. Mater. 2014, 26, 2268-2272.

(13) Park, H.; Lee, S. H.; Kim, F. S.; Choi, H. H.; Cheong, I. W.; Kim, J. H. Enhanced Thermoelectric Properties of PEDOT:PSS Nanofilms by a Chemical Dedoping Process. $J$. Mater. Chem. A 2014, 2, 6532-6539.

(14) Fan, Z.; Li, P.; Du, D.; Ouyang, J. Significantly Enhanced Thermoelectric Properties of PEDOT:PSS Films through Sequential Post-Treatments with Common Acids and Bases. Adv. Energy Mater. 2017, 7, 1602116.

(15) Yeon, C.; Yun, S. J.; Kim, J.; Lim, J. W. PEDOT:PSS Films with Greatly Enhanced Conductivity via Nitric Acid Treatment at Room Temperature and Their Application as Pt/TCOFree Counter Electrodes in Dye-Sensitized Solar Cells. Adv. Electron. Mater. 2015, 1, 1500121.

(16) Shi, H.; Liu, C.; Jiang, Q.; Xu, J. Effective Approaches to Improve the Electrical Conductivity of PEDOT:PSS: A Review. Adv. Electron. Mater. 2015, 1, 1500017.

(17) Gueye, M. N.; Carella, A.; Massonnet, N.; Yvenou, E.; Brenet, S.; Faure-Vincent, J.; 
Pouget, S.; Rieutord, F.; Okuno, H.; Benayad, A.; Demadrille, R.; Simonato, J. P. Structure and Dopant Engineering in PEDOT Thin Films: Practical Tools for a Dramatic Conductivity Enhancement. Chem. Mater. 2016, 28, 3462-3468.

(18) Kim, G.; Shao, L.; Zhang, K.; Pipe, K. P. Engineered Doping of Organic Semiconductors for Enhanced Thermoelectric Efficiency. Nat. Mater. 2013, 12, 719-723.

(19) Mengistie, D. A.; Ibrahem, M. A.; Wang, P. C.; Chu, C. W. Highly Conductive PEDOT:PSS Treated with Formic Acid for ITO-Free Polymer Solar Cells. ACS Appl. Mater. Interfaces 2014, 6, 2292-2299.

(20) Mengistie, D. A.; Chen, C. H.; Boopathi, K. M.; Pranoto, F. W.; Li, L. J.; Chu, C. W. Enhanced Thermoelectric Performance of PEDOT:PSS Flexible Bulky Papers by Treatment with Secondary Dopants. ACS Appl. Mater. Interfaces 2015, 7, 94-100.

(21) Massonnet, N.; Carella, A.; Jaudouin, O.; Rannou, P.; Laval, G.; Celle, C.; Simonato, J.-P. Improvement of the Seebeck Coefficient of PEDOT:PSS by Chemical Reduction Combined with a Novel Method for Its Transfer Using Free-Standing Thin Films. J. Mater. Chem. C 2014, 2, $1278-1283$.

(22) Bubnova, O.; Khan, Z. U.; Malti, A.; Braun, S.; Fahlman, M.; Berggren, M.; Crispin, X. Optimization of the Thermoelectric Figure of Merit in the Conducting Polymer Poly(3,4Ethylenedioxythiophene). Nat. Mater. 2011, 10, 429-433. 
(23) Myint, M. T. Z.; Hada, M.; Inoue, H.; Marui, T.; Nishikawa, T.; Nishina, Y.; Ichimura, S.; Umeno, M.; Kyaw, A. K. K.; Hayashi, Y. Simultaneous Improvement in Electrical Conductivity and Seebeck Coefficient of PEDOT:PSS by $\mathrm{N}_{2}$ Pressure-Induced Nitric Acid Treatment. RSC $A d v .2018,36563-36570$.

(24) Nardes, A. M.; Kemerink, M.; de Kok, M. M.; Vinken, E.; Maturova, K.; Janssen, R. A. J. Conductivity, Work Function, and Environmental Stability of PEDOT:PSS Thin Films Treated with Sorbitol. Org. Electron. physics, Mater. Appl. 2008, 9, 727-734.

(25) Zapata-Arteaga, O.; Dörling, B.; Perevedentsev, A.; Martín, J.; Reparaz, J. S.; CampoyQuiles, M. Closing the Stability-Performance Gap in Organic Thermoelectrics by Adjusting the Partial to Integer Charge Transfer Ratio. Macromolecules 2020, 53, 609-620.

(26) Yemata, T. A.; Zheng, Y.; Ko Kyaw, A. K.; Wang, X.; Song, J.; Chin, W. S.; Xu, J. Sodium Formaldehyde Sulfoxylate, an Ionic-Type, Water-Soluble Reducing Reagent to Effectively Improve Seebeck Coefficient of PEDOT:PSS Film. Org. Electron. 2020, 81, 105682.

(27) Yemata, T. A.; Kyaw, A. K. K.; Zheng, Y.; Wang, X.; Zhu, Q.; Chin, W. S.; Xu, J. Enhanced Thermoelectric Performance of Poly(3,4-ethylenedioxythiophene):Poly(4styrenesulfonate) (PEDOT:PSS) with Long-term Humidity Stability via Sequential Treatment with Trifluoroacetic Acid. Polym. Int. 2020, 69, 84-92.

(28) Mi, B. X.; Gao, Z. Q.; Cheah, K. W.; Chen, C. H. Organic Light-Emitting Diodes Using 3,6-Difluoro-2,5,7,7,8,8-Hexacyanoquinodimethane as p-Type Dopant. Appl. Phys. Lett. 2009, 
$94,073507$.

(29) Gwinner, M. C.; Pietro, R. Di; Vaynzof, Y.; Greenberg, K. J.; Ho, P. K. H.; Friend, R. H.; Sirringhaus, H. Doping of Organic Semiconductors Using Molybdenum Trioxide: A Quantitative Time-Dependent Electrical and Spectroscopic Study. Adv. Funct. Mater. 2011, 21, 1432-1441.

(30) Howells, C. T.; Saylan, S.; Kim, H.; Marbou, K.; Aoyama, T.; Nakao, A.; Uchiyama, M.; Samuel, I. D. W.; Kim, D. W.; Dahlem, M. S.; André, P. Influence of Perfluorinated Ionomer in PEDOT:PSS on the Rectification and Degradation of Organic Photovoltaic Cells. J. Mater. Chem. A 2018, 6, 16012-16028.

(31) Freeman, D. D.; Choi, K.; Yu, C. N-Type Thermoelectric Performance of Functionalized Carbon Nanotube-Filled Polymer Composites. PLoS One 2012, 7, e47822.

(32) Zhou, Y.; Fuentes-Hernandez, C.; Shim, J.; Meyer, J.; Giordano, A. J.; Li, H.; Winget, P.; Papadopoulos, T.; Cheun, H.; Kim, J.; Fenoll, M.; Dindar, A.; Haske, W.; Najafabadi, E.; Khan, T. M.; Sojoudi, H.; Barlow, S.; Graham, S.; Brédas, J. L.; Marder, S. R.; Kahn, A.; Kippelen, B. A Universal Method to Produce Low-Work Function Electrodes for Organic Electronics. Science (80-. ). 2012, 336, 327-332.

(33) Wang, J.; Fei, F.; Luo, Q.; Nie, S.; Wu, N.; Chen, X.; Su, W.; Li, Y.; Ma, C. Q. Modification of the Highly Conductive PEDOT:PSS Layer for Use in Silver Nanogrid Electrodes for Flexible Inverted Polymer Solar Cells. ACS Appl. Mater. Interfaces 2017, 9, 7834-7842. 
(34) Peng, X.; Hu, L.; Qin, F.; Zhou, Y.; Chu, P. K. Low Work Function Surface Modifiers for Solution-Processed Electronics: A Review. Adv. Mater. Interfaces 2018, 5, 1701404.

(35) Liu, J.; Wang, X.; Li, D.; Coates, N. E.; Segalman, R. A.; Cahill, D. G. Thermal Conductivity and Elastic Constants of PEDOT:PSS with High Electrical Conductivity. Macromolecules 2015, 48, 585-591.

(36) Fan, Z.; Ouyang, J. Thermoelectric Properties of PEDOT:PSS. Adv. Electron. Mater. 2019, $5,1800769$.

(37) Smith, P. M.; Su, L.; Gong, W.; Nakamura, N.; Reeja-Jayan, B.; Shen, S. Thermal Conductivity of Poly(3,4-Ethylenedioxythiophene) Films Engineered by Oxidative Chemical Vapor Deposition (OCVD). RSC Adv. 2018, 8, 19348-19352.

(38) Stavytska-Barba, M.; Kelley, A. M. Surface-Enhanced Raman Study of the Interaction of PEDOT: PSS with Plasmonically Active Nanoparticles. J. Phys. Chem. C 2010, 114, 6822-6830.

(39) Lucas, H.; Petitet, J. P. High Pressure Raman Spectroscopy of Nitric Acid. J. Phys. Chem. A 1999, 103, 8952-8958.

(40) Li, P.; Wang, G.; Cai, L.; Ding, B.; Zhou, D.; Hu, Y.; Zhang, Y.; Xiang, J.; Wan, K.; Chen, L.; Alameh, K.; Song, Q. High-Efficiency Inverted Polymer Solar Cells Controlled by the Thickness of Polyethylenimine Ethoxylated (PEIE) Interfacial Layers. Phys. Chem. Chem. Phys. 2014, 16, 23792-23799. 
(41) Zhou, Y.; Fuentes-Hernandez, C.; Shim, J.; Meyer, J.; Giordano, A. J.; Li, H.; Winget, P.; Papadopoulos, T.; Cheun, H.; Kim, J.; Fenoll, M.; Dindar, A.; Haske, W.; Najafabadi, E.; Khan, T. M.; Sojoudi, H.; Barlow, S.; Graham, S.; Bredas, J.-L.; Marder, S. R.; Kahn, A.; Kippelen, B. A Universal Method to Produce Low-Work Function Electrodes for Organic Electronics. Science (80-. ). 2012, 336, 327-332.

(42) Yurash, B.; Cao, D. X.; Brus, V. V.; Leifert, D.; Wang, M.; Dixon, A.; Seifrid, M.; Mansour, A. E.; Lungwitz, D.; Liu, T.; Santiago, P. J.; Graham, K. R.; Koch, N.; Bazan, G. C.; Nguyen, T.-Q. Towards Understanding the Doping Mechanism of Organic Semiconductors by Lewis Acids. Nat. Mater. 2019, 18, 1327-1334.

(43) Wu, F.; Li, P.; Sun, K.; Zhou, Y.; Chen, W.; Fu, J.; Li, M.; Lu, S.; Wei, D.; Tang, X.; Zang, Z.; Sun, L.; Liu, X.; Ouyang, J. Conductivity Enhancement of PEDOT:PSS via Addition of Chloroplatinic Acid and Its Mechanism. Adv. Electron. Mater. 2017, 3, 1700047.

(44) Wang, C.; Sun, K.; Fu, J.; Chen, R.; Li, M.; Zang, Z.; Liu, X.; Li, B.; Gong, H.; Ouyang, J. Enhancement of Conductivity and Thermoelectric Property of PEDOT:PSS via Acid Doping and Single Post-Treatment for Flexible Power Generator. Adv. Sustain. Syst. 2018, 2, 1800085.

(45) Lee, I.; Kim, G. W.; Yang, M.; Kim, T. S. Simultaneously Enhancing the Cohesion and Electrical Conductivity of PEDOT:PSS Conductive Polymer Films Using DMSO Additives. ACS Appl. Mater. Interfaces 2016, 8, 302-310.

(46) Mityashin, A.; Olivier, Y.; Van Regemorter, T.; Rolin, C.; Verlaak, S.; Martinelli, N. G.; 
Beljonne, D.; Cornil, J.; Genoe, J.; Heremans, P. Unraveling the Mechanism of Molecular Doping in Organic Semiconductors. Adv. Mater. 2012, 24, 1535-1539.

(47) Hosseini, E.; Ozhukil Kollath, V.; Karan, K. The Key Mechanism of Conductivity in PEDOT:PSS Thin Films Exposed by Anomalous Conduction Behaviour upon Solvent-Doping and Sulfuric Acid Post-Treatment. J. Mater. Chem. C 2020, 8, 3982-3990.

(48) Yan, F.; Parrott, E. P. J.; Ung, B. S.-Y.; Pickwell-MacPherson, E. Solvent Doping of PEDOT/PSS: Effect on Terahertz Optoelectronic Properties and Utilization in Terahertz Devices. J. Phys. Chem. C 2015, 119, 6813-6818.

(49) Liu, W.; Müller, L.; Ma, S.; Barlow, S.; Marder, S. R.; Kowalsky, W.; Köhn, A.; Lovrincic, R. Origin of the $\pi-\pi$ Spacing Change upon Doping of Semiconducting Polymers. $J$. Phys. Chem. C 2018, 122, 27983-27990.

(50) Emin, D. Enhanced Seebeck Coefficient from Carrier-Induced Vibrational Softening. Phys. Rev. B - Condens. Matter Mater. Phys. 1999, 59, 6205-6210.

(51) Patel, S. N.; Glaudell, A. M.; Kiefer, D.; Chabinyc, M. L. Increasing the Thermoelectric Power Factor of a Semiconducting Polymer by Doping from the Vapor Phase. ACS Macro Lett. 2016, 5, 268-272.

(52) Emin, D. Vibrational Contribution to the Seebeck Coefficient of Bipolaronic Carriers in Boron Carbides. Phys. status solidi 1998, 205, 385-390. 


\section{Table of Contents (TOC)}

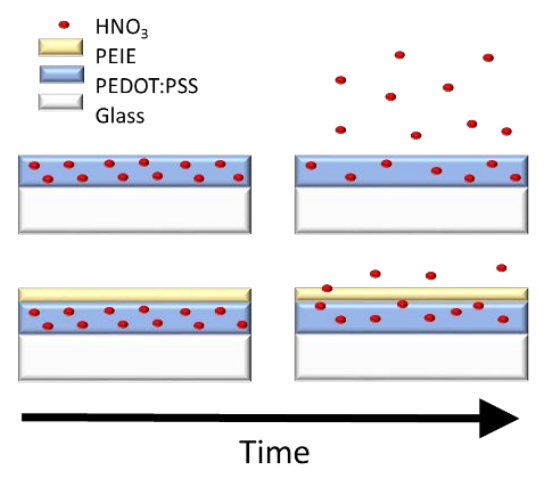

